\title{
Successful prediction of dominance in convict cichlids, Cichlasoma nigrofasciatum
}

\author{
PAUL M. BRONSTEIN \\ University of Michigan, Flint, Michigan \\ and \\ PAUL F. BRAIN \\ University College of Swansea, Swansea, Wales
}

\begin{abstract}
In each of seven pairs of size-matched convict cichlids, the first animal to bite became the dominant fighter.
\end{abstract}

Studies of combat in dyads of aggressive fish provide some empirical support for the position that, in some cases, agonistic behavior occurring at the start of fighting is both asymmetric and highly predictive of animals' subsequent fighting behavior (see Huntingford \& Turner, 1987, pp. 284-287). For instance, using pairs of convict cichlids, Figler and his colleagues have shown that the first fish to bite is typically the victor or dominant animal in the fight (Figler \& Einhorn, 1983; Figler \& Evensen, 1979; Figler \& Peeke, 1978; Figler, Wazlavek, Spencer, \& Gussio, 1985). However, there are several potential problems for interpreters of those cichlid studies. The first-bite effect is not always statistically reliable, and the effect generally has been found during assessments either of drugs or of familiarity with environmental cues on aggressive behavior. Thus, asymmetric contests in this species may occur only in particular situations.

Also, Figler's animal-management procedures sometimes have involved returning fish to a community tank following one study, with animals then made available for use in subsequent experiments (M. H. Figler, personal communication, August 1984). This animal-procurement technique, especially when combined with the procedure of matching pairs of combatants on the basis of body size, represents a potential bias that might exaggerate the positive correlation between the first bite and the identity of the fish that eventually becomes dominant. For example, the same animals could be paired repeatedly and may carry some memory of their prior victories and defeats into subsequent experiments (see Peeke, Herz, \& Gallagher, 1971). Furthermore, since some of the same fish may have been paired repeatedly, it is impossible to assess the reliability of this first-bite effect just by summing the results

This research was supported by grants to the first author from the Rackham Foundation and from the Faculty Development Committee of the University of Michigan-Flint. We thank D. O. F. Skibinski for providing the animals. Correspondence should be addressed to Paul $\mathbf{M}$. Bronstein, Department of Psychology, University of Michigan-Flint, Flint, MI 48502-2186. reported in the separate, but potentially nonindependent, studies. The current experiment was an attempt to eliminate these sources of possible ambiguity and to determine whether the outcomes of agonistic contests in Cichlasoma nigrofasciatum could be predicted by behavior occurring at or near the start of fighting.

\section{METHOD}

Convict cichlids were purchased from local suppliers in Swansea, Wales, and maintained in a mixed-sex group $(19<N<60)$ in an opaque plastic community tank (40 liter) for approximately 12 months. Lights were on from 0630 to $2030 \mathrm{~h}$ local time; during all phases of the study, food (Ewos Baker Trout Pellets) was provided daily. Fish lived and were tested in aerated tap water that was aged at least $24 \mathrm{~h}$ and kept at $23^{\circ}-26^{\circ} \mathrm{C}$.

The subjects were netted, and their standard lengths (from snout to caudal peduncle) were measured (range $=55.6-102.2 \mathrm{~mm}$ ). They were then rehoused individually for 5 weeks. Lighting was then provided from 0900 to $2100 \mathrm{~h}$ local time; each animal was kept in half of a $61 \times 20 \times 20 \mathrm{~cm}$ all-glass tank. These tanks contained an air stone and a substrate of undyed gravel, and they were divided at the midpoints of their long walls by double thicknesses of fine, white netting fixed to walls and floor with silicon aquarium cement. Thus, the subjects, although they could not touch each other, were provided with both chemical and visual stimulation from a conspecific during this period of relative isolation. In addition, most fish could see other animals through the clear glass sides of their tanks.

Eight animals were size-matched to partners that were not more than $6 \%$ different from their standard lengths; the median size difference within dyads was $2 \%$. The fish were housed such that the partners could not see each other. At testing, the members of a pair were netted separately and placed simultaneously into a tank $(41 \times 20 \times 20 \mathrm{~cm})$ having aeration and undyed gravel flooring. This test aquarium was all glass, except for one short wall composed of a double thickness of white netting. The animals were tested in water in which conspecifics had swum.

For their first 15 min together (adaptation), the partners were separated by a clear glass barrier. This partition was then removed to permit $30 \mathrm{~min}$ of physical contact. Sessions were videotaped and later replayed, with attacks scored for each animal. Two separate behaviors constituted this general category: (1) Butting-one fish ramming the other with its head. This act lasted less than $0.2 \mathrm{sec}$ and was typically accompanied by visible biting movements. The fish receiving such attacks nearly always escaped or, if trapped by the wall(s) of its tank, remained immobile. Out of a total of 308 attacks, only 2 resulted in nearly simultaneous counterattack. (2) Charging-one animal rapidly swimming at its partner. This maneuver was essentially the butting act accompanied by effective, anticipatory escape by the target animal. 


\section{RESULTS AND DISCUSSION}

One pair of animals remained immobile throughout testing and was excluded from the analysis. Five of the remaining seven dyads were characterized by only one of the fish doing any attacking, with opponents always retreating. In one further pair, the dominant animal exhibited 35 attacks, while its partner attacked only once; and finally, there was a pair with one fish attacking 35 times, answered by two counterattacks from its opponent. The dominant, attacking fish had a median of 35 attacks (range $=4-143$ ); the subordinates all stopped attacking after at most $6.5 \mathrm{~min}$ with their opponents. Also, only three of the contests were won by the larger fish of each pair, and this lack of a body-size effect confirms the adequacy of size-matching procedures. Finally, the first attack (always a butt) had a median latency of 109 sec (range = 55-220 sec) and was always made by the dominant, attacking animal $(p<.02)$. Thus, convict cichlids in aggressive encounters do provide human observers (and, potentially, conspecific opponents) with accurate information about their subsequent agonistic behavior at the moment an initial bite is made. Persistent attack-hence, the control of space (i.e., the attainment of dominant status)-follows reliably upon the relatively rapid onset of biting. The potential problems with earlier data on this topic appear not to have exaggerated this effect; fish can be readily classified into attackers and nonattackers after only a few minutes of observation. Attackers (i.e., first biters) also bite opponents most persistently and, thus, become dominant.

\section{REFERENCES}

Figler, M. H., \& EINHORN, D. M. (1983). The territorial prior residence effect in convict cichlids (Cichlasoma nigrofasciatum Gunther): Temporal aspects of establishment and retention, and proximate mechanisms. Behaviour, 85, 157-183.

Figler, M. H., EVENSEN, J. (1979). Experimentally produced prior residence effect in male convict cichlids: The role of initial proximity to territorial markers. Bulletin of the Psychonomic Society, 13, 130-132.

Figler, M. H., PeEke, H. V. S. (1978). Alcohol and the prior residence effect in male convict cichlids (Cichlasoma nigrofasciatum). Aggressive Behavior, 4, 125-132.

Figler, M. H., Wazlavek, B. E., Spencer, F. P., \&ussio, R. P. (1985). The influence of chemical cues on the territorial prior residence effect in convict cichlids (Cichlasoma nigrofasciatum Gunther). Aggressive Behavior, 11, 207-216.

Huntingford, F. A., \& TURner, A. (1987). Animal conflict. London: Chapman \& Hall.

Peeke, H. V. S., Herz, M. J., \& Gallagher, J. E. (1971). Changes in aggressive interaction in adjacently territorial convict cichlids (Cichlasoma nigrofasciatum): A study of habituation. Behaviour, 40, 43-54.

(Manuscript received May 10, 1991.) 